\section{Factores asociados a inactividad física en Chile: resultados de la Encuesta Nacional de Salud 2009-2010}

\author{
XIMENA DÍAZ-MARTÍNEZ ${ }^{1}$, ALEXIS GARRIDO², \\ MARÍA ADELA MARTÍNEZ ${ }^{3}$, ANA MARÍA LEIVA ${ }^{4}$, \\ CRISTIAN ÁLVAREZ ${ }^{5}$, RODRIGO RAMÍREZ-CAMPILLO ${ }^{5}$, \\ CARLOS CRISTI-MONTERO ${ }^{6}$, FERNANDO RODRÍGUEZ ${ }^{6}$, \\ CARLOSSALAS-BRAVO ${ }^{7}$, ELIANADURÁN $^{8}$,ANAMARÍALABRAÑA ${ }^{8}$, \\ PEDRO VALDIVIA-MORAL ${ }^{9}$, MARÍA LUISA ZAGALAZ ${ }^{10}$, \\ NICOLÁS AGUILAR-FARÍAS ${ }^{11}$, CARLOS A. CELIS-MORALES ${ }^{12,13}$
}

\section{Correlates of physical inactivity: Findings from the Chilean National Health Survey 2009-2010}

Background: Physical inactivity is an important risk factor for cardiovascular diseases. Aim: To identify factors associated with physical inactivity in Chilean adults. Material and Methods: Participants from the Chilean Health Survey (5,133 individuals) were included in this study. Physical activity was measured using the Global physical activity questionnaire (GPAQ). Physical inactivity was defined as $<600$ MET. min.week ${ }^{-1}$ of moderate to vigorous intensity physical activity. Logistic regression was used to identify correlates of physical inactivity. Results: The main finding of this study were that women (odds ratio (OR): 1.51 [95\% confidence intervals (CI): 1.32-1.72]) were more likely to be inactive compared to men and that older adults were more likely to be inactive than their younger or middle age counterparts (OR: 3.06 [95\% CI: 2.45-3.82]). Other correlates of physical inactivity were individuals with obesity (OR: 1.43 [95\% CI: 1.20-1.70]), diabetes (OR: 1.96 [95\% CI: 1.61-2.38]), hypertension (OR: 1.72 [95\% CI: 1.50-1.97]), metabolic syndrome (OR: 1.42 [95\% IC: 1.18-1.70]), a low education level (OR: 1.26 [95\% CI: 1.06-1.49]) and higher levels of sedentary behavior (OR: 2.77 [95\% CI: 2.36-3.25]). Conclusions: Women, older people and sedentary subjects with chronic non-transmissible conditions are at higher risk of being physically inactive.

(Rev Med Chile 2017; 145: 1259-1267)

Key words: Cardiovascular Diseases; Physical activiy; Obesity; Risk Factors; Sedentary Lifestyle.
'Grupo de Investigación Calidad de Vida, Departamento de Educación, Universidad del Biobío. Chillán, Chile.

${ }^{2}$ Departamento de Educación Física, Universidad San Sebastián, Concepción, Chile.

${ }^{3}$ Instituto de Farmacia, Facultad de Ciencias, Universidad Austral de Chile, Valdivia, Chile. ${ }^{4}$ Instituto de Anatomía, Histología y Patología, Facultad de Medicina, Universidad Austral de Chile, Valdivia, Chile.

${ }^{5}$ Departamento de Ciencias de la Actividad Física, Universidad de Los Lagos, Osorno, Chile.

${ }^{6}$ Grupo IRyS, Escuela de Educación Física, Pontificia Universidad Católica de Valparaíso Valparaíso, Chile.

${ }^{7}$ Departamento de Educación Física, Facultad de Educación, Universidad de Concepción. Concepción, Chile.

${ }^{8}$ Departamento de Nutrición y Dietética, Facultad de Farmacia, Universidad de Concepción, Concepción, Chile.

'Universidad de Granada (España). Grupo de Investigación del PAIDI, HUM653, Innovación Didáctica en Actividad Física (IDAF).

${ }^{10}$ Universidad de Jaén (España). Grupo de Investigación del PAIDI, HUM653, Innovación Didáctica en Actividad Física (IDAF).

"Departamento de Educación Física, Deporte y Recreación, Universidad de La Frontera, Temuco, Chile.

${ }^{12}$ BHF Glasgow Cardiovascular Research Centre, Institute of Cardiovascular and Medical Science, University of Glasgow, Glasgow, United Kingdom, G2 8AT. ${ }^{13}$ Centro de Investigaciones en Fisiología Integrada y Salud, Universidad Mayor, Santiago, Chile.

Recibido el 4 de noviembre de 2016, aceptado el 28 de agosto de 2017.

\section{Correspondencia a:}

Dr. Carlos Celis-Morales

BHF Glasgow Cardiovascular Research Centre, Institute of Cardiovascular and Medical Sciences, College of Medical, Veterinary and LifeSciences, University of Glasgow, Glasgow G12 8TA, United Kingdom.

carlos.celis@glasgow.ac.uk

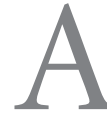

nivel mundial la falta de actividad física (AF) se ha convertido en uno de los factores de riesgo más importante en el desarrollo de enfermedades crónicas no transmisibles ${ }^{1-3}$. Ser inactivo ( $<150$ min de AF de intensidad moderada o vigorosa a la semana o su equivalente realizar $<600 \mathrm{METs} / \mathrm{min} / \mathrm{semana})$, es causante de un $6 \%$ a $10 \%$ del riesgo de sufrir enfermedad coronaria, 
hipertensión, diabetes mellitus tipo 2, y cáncer de mama y de colon ${ }^{1}$. Más aún, 9\% de las muertes prematuras está asociado con la inactividad física, esto equivale a 5,3 millones de muertes por año ${ }^{1}$. Se estima que el reducir la inactividad física entre un $10 \%$ a $25 \%$ permitiría disminuir a nivel mundial, el número de muertes prematuras entre 0,5 y 1,3 millones por año ${ }^{1}$.

A pesar que existe suficiente evidencia científica que confirma los beneficios de la práctica regular de $\mathrm{AF}^{1,3-5}$, actualmente $31,1 \%$ de la población adulta a nivel mundial no cumple con las recomendaciones de $\mathrm{AF}^{6}$, mientras que en Chile $27,1 \%$ de la población reportó ser físicamente inactiva ${ }^{7}$. Se plantea que los niveles de inactividad física han aumentado debido al incremento en urbanización, como también la modernización de la sociedad, lo cual incluye, una dependencia mayor de sistemas motorizados de transporte, tecnología (aumento del número de televisores y computadores asociados a mayor tiempo sentado), y también períodos prolongados de trabajo de tipo sedente, principalmente trabajos de oficina y comercio, donde los trabajadores pasan la mayor parte de su jornada sentados ${ }^{8}$. Si bien, estos son algunos de los factores que podrían asociarse al aumento de los niveles de inactividad física, en Chile, se desconoce qué factores sociales, demográficos o estilos de vida se asocian con ser físicamente inactivo. Estudios previos realizados en población chilena han comprobado la relación entre niveles de actividad física y factores de riesgo cardiovascular $^{9-14}$, no obstante, estos estudios no han identificado que factores se asocian a ser físicamente inactivo o si existen diferencias en factores asociados a inactividad entre género. Identificar estos factores podría a ser un paso importante para implementar intervenciones personalizadas como también orientar los esfuerzos gubernamentales a aquellos sectores de la población que presentan un mayor riesgo de ser físicamente inactivos. En consecuencia, el objetivo de este estudio fue identificar qué factores sociales, demográficos, estilos de vida y de salud de la población chilena se asocian con inactividad física.

\section{Materiales y Métodos}

\section{Diseño del estudio}

La muestra seleccionada comprende todos los participantes de la Encuesta Nacional de Salud
(ENS) 2009-2010 desarrollada entre octubre del año 2009 y septiembre de 20107. La ENS 2009-10 corresponde a un estudio de prevalencia realizado en hogares en una muestra nacional, probabilística, estratificada y multietápica de 5,412 personas mayores de 15 años con representatividad nacional, regional, y área urbana/rural. Un total de 5,133 participantes con información disponible de AF fueron incluidos en este estudio. Los participantes firmaron un consentimiento informado ${ }^{7}$. El protocolo de estudio fue aprobado por el Comité de Ética de Investigación de la Escuela de Medicina de la Pontificia Universidad Católica de Chile.

\section{Mediciones antropométricas y metabólicas}

Las muestras de sangre de los participantes en ayuno, fueron obtenidas por una enfermera entrenada siguiendo protocolos estandarizados a nivel nacional ${ }^{7}$. Los marcadores metabólicos de glicemia, perfil lipídico (triglicéridos, colesterol HDL, colesterol LDL, colesterol total) y presión arterial fueron medidos con métodos estandarizados y previamente descritos en la ENS ${ }^{7}$. Los puntos de corte utilizados para la detección de enfermedades cardiovasculares fueron los siguientes: hipertensión (PAS $\geq 140$ o $\mathrm{PAD} \geq 90 \mathrm{mmHg}$ ), diabetes (glicemia en ayuno $\geq 126 \mathrm{mg}^{-\mathrm{dl}^{-1}}$ ). El síndrome metabólico fue determinado utilizando el criterio de la IDF-ATP ${ }^{7,15}$, que requiere al menos 3 de los siguientes 5 criterios para su clasificación: presión arterial $(>130 / 85 \mathrm{mmHg})$, circunferencia cintura elevada ( $>88 \mathrm{~cm}$ en hombres $\mathrm{o} \geq 83 \mathrm{~cm}$ en mujeres), colesterol HDL ( $<40 \mathrm{mg} / \mathrm{dl}$ en hombres $\mathrm{o}<50$ en mujeres), glicemia elevada $(>100 \mathrm{mg}$. $\left.\mathrm{dl}^{-1}\right)$, triglicéridos elevados $(>150 \mathrm{mg} / \mathrm{dl})$. El índice de masa corporal (IMC) fue utilizado para determinar el estado nutricional según los criterios de la OMS (Normal: < 25,0; Sobrepeso: 25,0-29,9; Obeso: $\geq 30,0)$. El perímetro de cintura sugerido para población chilena fue utilizado para definir la clasificación de obesidad central ( $>87 \mathrm{~cm}$ para hombres y $>82 \mathrm{~cm}$ para mujeres $)^{7}$.

Las variables socio-demográficas (edad, sexo, nivel educacional, ingreso económico) y datos asociados con estilos de vida, como el tabaquismo, consumo de frutas, verduras y alcohol se obtuvieron mediante la aplicación de cuestionarios validados en la ENS ${ }^{7}$. El estado de bienestar y salud fue auto-reportado a través de la aplicación de cuestionarios y los participantes podrían seleccionar las siguientes opciones (buena, regular 
o mala). Para nivel de ingreso se creó una variable categórica utilizando los siguientes puntos de corte: Bajo: <250,000, Medio: 250,000-450,000, Alto: $>450.000$ mil pesos. Para consumo de alcohol y consumo de frutas y verduras se crearon terciles de consumo diario utilizando los siguientes puntos de corte para alcohol (Bajo: < 18,7, Medio: 18,7-44, Alto: $>44 \mathrm{~g} /$ día) y frutas y verduras (Bajo: $<145$, Medio: 145-240, Alto: >240 g/día).

\section{Clasificación de actividad física}

El tiempo destinado a AF relacionada al transporte (ej. caminar, andar en bicicleta), y actividades de intensidad moderada o vigorosa realizadas en el tiempo libre y en el trabajo, fueron determinadas según la guía de análisis de GPAQ (Global Physical Activity Questionnaire v2) ${ }^{16}$. Para determinar los niveles de AF total, las variables fueron expresadas en METs (Metabolic-energy-equivalents) específicos a cada intensidad de AF (4 METs para AF de transporte y AF de intensidad moderada y 8 METs para AF de intensidad vigorosa). Para esta estimación, los minutos de $\mathrm{AF}$ reportados para transporte, $\mathrm{AF}$ moderada y $\mathrm{AF}$ vigorosa fueron multiplicados por sus equivalentes en METs. Se consideró como punto de corte para inactividad física un gasto energético $<600$ METs/min/semana, según las recomendación de la OMS y especificaciones en la guía de análisis de GPAQ ${ }^{16,17}$. Los niveles de conducta sedentaria fueron determinados mediante el auto-reporte de tiempo destinado a actividades que involucren estar sentado o reclinado durante el tiempo libre o de trabajo. Los terciles de conductas sedentarias fueron definidos con los siguientes puntos de corte: Bajo: $<2$, Medio: 2-4, Alto: $>4$ h/día).

\section{Análisis estadístico}

Las diferencias entre niveles de actividad física (activo versus inactivo) fueron determinadas con t-test para variables continuas con distribución normal y Mann-Whitney test para variables que no cumplían con una distribución normal. $\chi^{2}$ fue utilizado para variables categóricas. La asociación entre inactividad física y variables de interés se determinó mediante análisis de regresión logística. Estos análisis fueron ajustados por edad, sexo, tabaquismo, nivel educacional, ingreso económico e IMC. Estas variables fueron seleccionadas para ajustar los modelos estadísticos debido a su asociación con la variable de salida o "outcome" (inactividad física) como también así las variables independientes incluidas en los análisis.

Los datos para estos análisis fueron presentados como Odds ratio (OR) y sus respectivos intervalos de confianza de 95\% (95\% IC). Las variables continuas fueron transformadas a terciles (Bajo, Medio, Alto), con el fin de establecer una asociación entre terciles de ingreso, niveles de conducta sedentaria, consumo de alcohol, frutas y verduras con el riesgo de ser físicamente inactivo.

Para todos los análisis se utilizó el módulo de muestras complejas del programa STATA SE v14 y todos los resultados fueron estimados utilizando muestras expandidas según la ENS 2009-20107. El nivel de significancia fue definido como $\mathrm{p}<0,05$.

\section{Resultados}

Las características de la población estudiada según niveles de actividad física son presentadas en la Tabla 1. En resumen, los participantes clasificados como físicamente inactivos son mayoritariamente mujeres, tienen un mayor promedio de edad, nivel de escolaridad básica, una mayor prevalencia de obesidad (IMC $\geq 30,0$ ), y obesidad central. En relación a los estilos de vida, las personas físicamente inactivas destinan más tiempo a actividades de tipo sedente. En relación a la prevalencia de enfermedades, el grupo físicamente inactivo presenta una mayor prevalencia de diabetes, hipertensión y síndrome metabólico.

En la Figura 1 se presentan los factores socio-demográficos, de estilos de vida y salud asociados a la inactividad física. Estos resultados revelan que las mujeres tienen una mayor probabilidad de ser inactivas en comparación a los hombres. Las personas mayores de 65 años presentan una mayor probabilidad de ser inactivas en comparación con aquellos menores a 25 años de edad (OR: 3,06 [95\% IC: 2,45-3,82]). Otros factores que se asocian a una mayor probabilidad de ser inactivo son personas con obesidad, o con obesidad central, individuos que auto reportan tener un nivel de salud regular o mala, personas con diabetes, hipertensión, síndrome metabólico y niveles medios o altos de tiempo destinado a estar sentado (Figura 1). Los factores asociados a una menor probabilidad de ser inactivo fueron personas que reportaron ser ex-fumadoras o fumadoras, personas con niveles educacionales 
Tabla 1. Características de la población chilena según recomendación de actividad física

\begin{tabular}{|c|c|c|c|}
\hline Variables & Físicamente activo & Físicamente inactivo & Valor p \\
\hline \multicolumn{4}{|l|}{ Socio-demográficas } \\
\hline n & 3.917 & 1.216 & \\
\hline \multicolumn{4}{|l|}{$\mathrm{Sexo}^{\neq}$} \\
\hline $\begin{array}{l}\text { Mujeres } \\
\text { Hombres }\end{array}$ & $\begin{array}{l}57,2(55,7 ; 58,8) \\
42,7(41,1 ; 44,2)\end{array}$ & $\begin{array}{l}66,9(64,2 ; 69,5) \\
33,1(30,4 ; 35,7)\end{array}$ & $<0,0001$ \\
\hline Edad (años)* & $44,5(43,9 ; 45,1)$ & $52,5(51,3 ; 53,6)$ & $<0,0001$ \\
\hline \multicolumn{4}{|l|}{ Grupo etario ${ }^{\ddagger}$} \\
\hline $\begin{array}{l}<25 \text { años } \\
25-44 \text { años } \\
45-64 \text { años } \\
\geq 65 \text { años }\end{array}$ & $\begin{array}{l}16,2(15,1 ; 17,4) \\
34,6(33,1 ; 36,1) \\
33,9(32,5 ; 35,4) \\
15,1(14,1 ; 16,3)\end{array}$ & $\begin{array}{l}11,5(9,8 ; 13,4) \\
26,5(24,1 ; 29,0) \\
29,2(26,731,8) \\
32,8(30,3 ; 35,5)\end{array}$ & $<0,00001$ \\
\hline \multicolumn{4}{|l|}{ Zona geográfica } \\
\hline $\begin{array}{l}\text { Rural } \\
\text { Urbano }\end{array}$ & $\begin{array}{l}14,9(13,8 ; 16,0) \\
85,1(83,9 ; 86,1)\end{array}$ & $\begin{array}{l}14,3(12,4 ; 16,4) \\
85,(83,6 ; 87,5)\end{array}$ & 0,619 \\
\hline \multicolumn{4}{|l|}{ Nivel educacional ${ }^{\ddagger}$} \\
\hline Básica & $23,7(22,4 ; 25,1)$ & $36,0(33,3 ; 38,7)$ & \\
\hline Media & $57,3(55,7 ; 58,8)$ & $45,9(43,1 ; 48,7)$ & $<0,0001$ \\
\hline Técnico Universitaria & $19,0(17,8 ; 20,2)$ & $18,1(16,0 ; 20,3)$ & \\
\hline \multicolumn{4}{|l|}{ Nivel de ingreso ${ }^{\ddagger}$} \\
\hline $\begin{array}{l}\text { Bajo }(<250.000 \text { mil pesos }) \\
\text { Medio }(250.000-450.000 \text { mil pesos })\end{array}$ & $\begin{array}{l}55,2(53,6 ; 56,8) \\
33,7(32,2 ; 35,3)\end{array}$ & $\begin{array}{l}58,4(55,6 ; 61,2) \\
31,3(28,7 ; 34,0)\end{array}$ & 0,137 \\
\hline Alto $(<450.000$ mil pesos) & $10,3 \quad(8,7 ; 12,2)$ & $10,3 \quad(8,7 ; 12,2)$ & \\
\hline \multicolumn{4}{|l|}{ Antropométricas } \\
\hline Peso $(\mathrm{kg})^{*}$ & $71,7(71,2 ; 72,1)$ & $71,8(70,9 ; 72,7)$ & 0,833 \\
\hline $\operatorname{IMC}\left(\mathrm{kg} / \mathrm{m}^{2}\right)^{*}$ & $27,7(27,5 ; 27,8)$ & $28,5(28,1 ; 28,8)$ & $<0,0001$ \\
\hline $\begin{array}{l}\text { Estado Nutricional IMC }{ }^{\ddagger} \\
<18,5 \mathrm{~kg} / \mathrm{m}^{2} \\
18,5-24,9 \mathrm{~kg} / \mathrm{m}^{2} \\
25,0-29,9 \mathrm{~kg} / \mathrm{m}^{2} \\
\geq 30,0 \mathrm{~kg} / \mathrm{m}^{2}\end{array}$ & $\begin{array}{c}1,8 \quad(1,4 ; 2,2) \\
29,6(28,2 ; 31,2) \\
41,2(39,6 ; 42,8) \\
27,3(25,9 ; 28,8)\end{array}$ & $\begin{array}{l}1,3 \quad(0,7 ; 2,1) \\
26,5(24,0 ; 29,2) \\
37,3(34,4 ; 40,1) \\
34,9(32,1 ; 37,8)\end{array}$ & $<0,0001$ \\
\hline Perímetro de cintura $(\mathrm{cm})^{*}$ & $95,7(95,3 ; 96,1)$ & $98,5(97,6 ; 99,3)$ & $<0,0001$ \\
\hline Obesidad central $\left.\right|^{\ddagger}$ & $67,8(66,2 ; 69,2)$ & $75,5(72,9 ; 77,9)$ & $<0,0001$ \\
\hline \multicolumn{4}{|l|}{ Estilos de vida } \\
\hline Actividad física total (MET/min/semana) $)^{\neq}$ & $6.480(2.460 ; 13.200)$ & $0 \quad(0 ; 120)$ & $<0,0001$ \\
\hline Actividad física de transporte (min/día) & $30 \quad(8,6 ; 75)$ & $0 \quad(0 ; 0)$ & $<0,0001$ \\
\hline Actividad física moderada (min/día) ${ }^{\neq}$ & $66,4(0 ; 240)$ & $0 \quad(0 ; 0)$ & $<0,0001$ \\
\hline Actividad física vigorosa (min/día) ${ }^{\neq}$ & $0 \quad(0 ; 42,9)$ & $0 \quad(0 ; 0)$ & $<0,0001$ \\
\hline Tiempo sedente $(\mathrm{h} / \mathrm{dí})^{\neq}$ & $2,2(1,3 ; 4)$ & $4 \quad(2 ; 6)$ & $<0,0001$ \\
\hline Consumo de frutas y vegetales (g/día)* & $216,9(212 ; 221)$ & $208,9(200 ; 217)$ & 0,103 \\
\hline Consumo de alcohol (g/día)* & $53,5(48,9 ; 58,4)$ & $50,6(40,8 ; 60,3)$ & 0,600 \\
\hline Consumo de sal (g/día)* & $9,71(9,6 ; 9,8)$ & $9,89(9,6 ; 10,1)$ & 0,147 \\
\hline \multicolumn{4}{|l|}{ Tabaquismo } \\
\hline $\begin{array}{l}\text { Nunca } \\
\text { Ex-fumador } \\
\text { Fumador }\end{array}$ & $\begin{array}{l}39,4(37,9 ; 41,0) \\
23,8(22,5 ; 25,2) \\
36,7(35,2 ; 38,2)\end{array}$ & $\begin{array}{l}47,4(44,7 ; 50,3) \\
24,1(21,7 ; 26,5) \\
28,4(26,0 ; 31,1)\end{array}$ & $<0,0001$ \\
\hline \multicolumn{4}{|l|}{ Enfermedades y salud } \\
\hline Diabetes mellitus tipo $2^{\ddagger}$ & $9,8 \quad(8,9 ; 10,9)$ & $17,6(15,4 ; 20,0)$ & $<0,0001$ \\
\hline Hipertensión arterial ${ }^{\ddagger}$ & $29,4(27,9 ; 30,9)$ & $41,8(38,9 ; 44,7)$ & $<0,0001$ \\
\hline Síndrome metabólico ${ }^{\ddagger}$ & $33,7(31,6 ; 35,9)$ & $42,0(38,2 ; 45,9)$ & $<0,0001$ \\
\hline \multicolumn{4}{|l|}{ Auto reporte de bienestar y salud ${ }^{\ddagger}$} \\
\hline $\begin{array}{l}\text { Buena } \\
\text { Regular } \\
\text { Mala }\end{array}$ & $\begin{array}{l}2,6(2,1 ; 3,1) \\
30,0(28,5 ; 31,4) \\
67,5(66,0 ; 68,9)\end{array}$ & $\begin{array}{l}5,0 \quad(3,9 ; 6,4) \\
34,4(31,8 ; 37,1) \\
60,6(57,8 ; 63,2)\end{array}$ & $<0,0001$ \\
\hline
\end{tabular}

Datos presentados como promedio para variables continuas $\left(^{*}\right)$ y cómo $\%$ para variables categóricas $\left({ }^{*}\right)$, ambos fueron presentados con sus respectivos intervalos de confianza del $95 \%$. Variables que no presentaban una distribución normal fueron presentadas como mediana y sus respectivos rangos de inter cuartil ${ }^{*}$. Diferencias entre variables continuas fueron determinadas con t-test para variables continuas con distribución normal y con Mann-Whitney test para variables sin distribución normal. Para variables categóricas se utilizó $\chi^{2}$. Se consideró como punto de corte para inactividad física un gasto energético < $600 \mathrm{METs/}$ $\mathrm{min} / \mathrm{semana}$, según las recomendación de la OMS y especificaciones en la guía de análisis de GPAQ. 
equivalente a educación media o educación técnico superior, personas con niveles de ingreso equivalentes al tercil medio, y personas ubicadas en el tercil alto de consumo de frutas y verduras. No se encontró asociación entre zona geográfica, consumo de alcohol y el riesgo de ser inactivo. Resultados similares fueron encontrados cuando

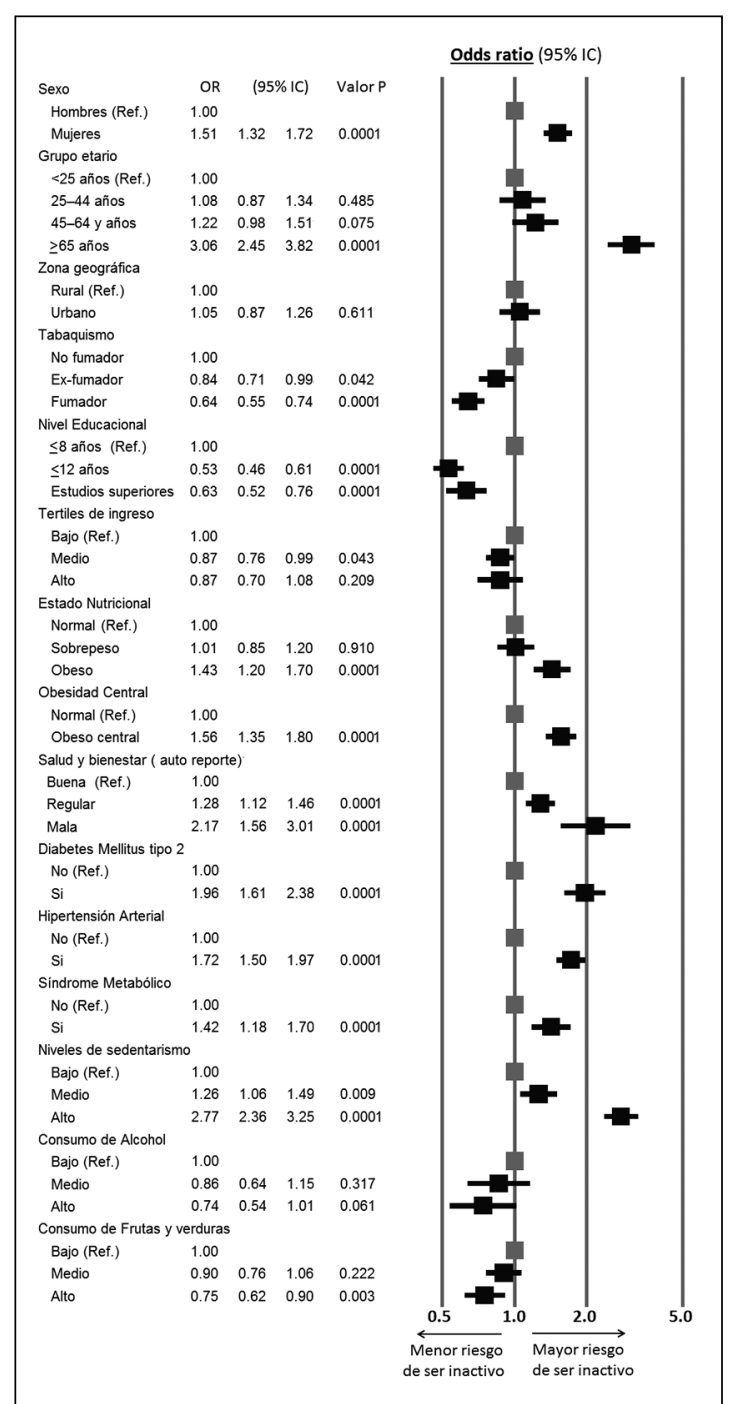

Figura 1 . Factores asociados a inactividad fisica en hombres y mujeres de la población chilena. Datos presentados como odds ratio y sus respectivos intervalos de confianza de $95 \%$. Todos los análisis fueron ajustados por edad, sexo, educación, zona geográfica, tabaquismo e IMC, a excepción de cuando estas variables fueron utilizadas como variable independente en el modelo estadístico. El grupo de referencia utilizado para cada variable se señala con (Ref.). los análisis fueron estratificados para hombres (Figura 2) y mujeres (Figura 3) a excepción de la asociación entre zona geográfica y probabilidad de ser inactivo. Los hombres que viven en zonas urbanas presentan una mayor probabilidad de ser inactivos en comparación a aquellos hombres que viven en zonas rurales (Figura 2).

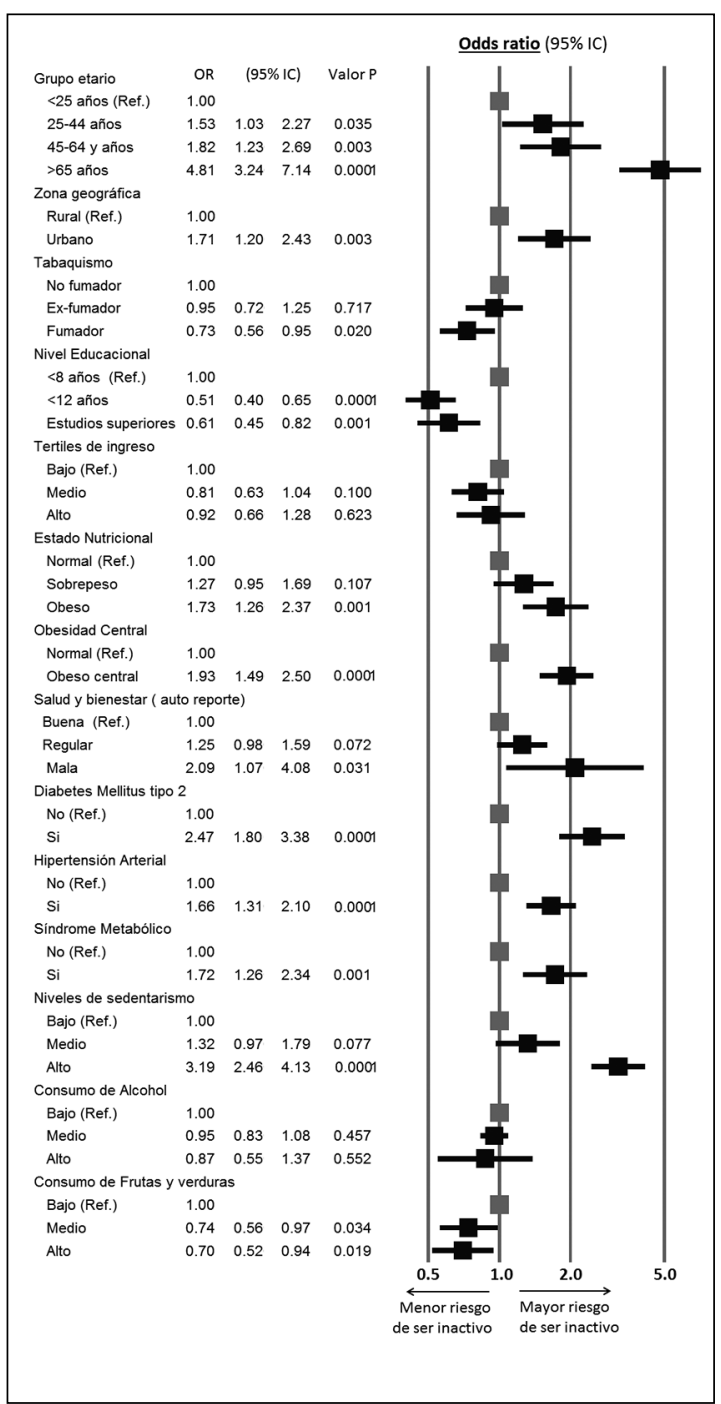

Figura 2. Factores asociados a inactividad fisica en hombres de la población chilena. Datos presentados como odds ratio y sus respectivos intervalos de confianza de $95 \%$. Todos los análisis fueron ajustados por edad, educación, zona geográfica, tabaquismo e IMC, a excepción de cuando estas variables fueron utilizadas como variable independente en el modelo estadístico. El grupo de referencia utilizado para cada variable se señala con (Ref.). 


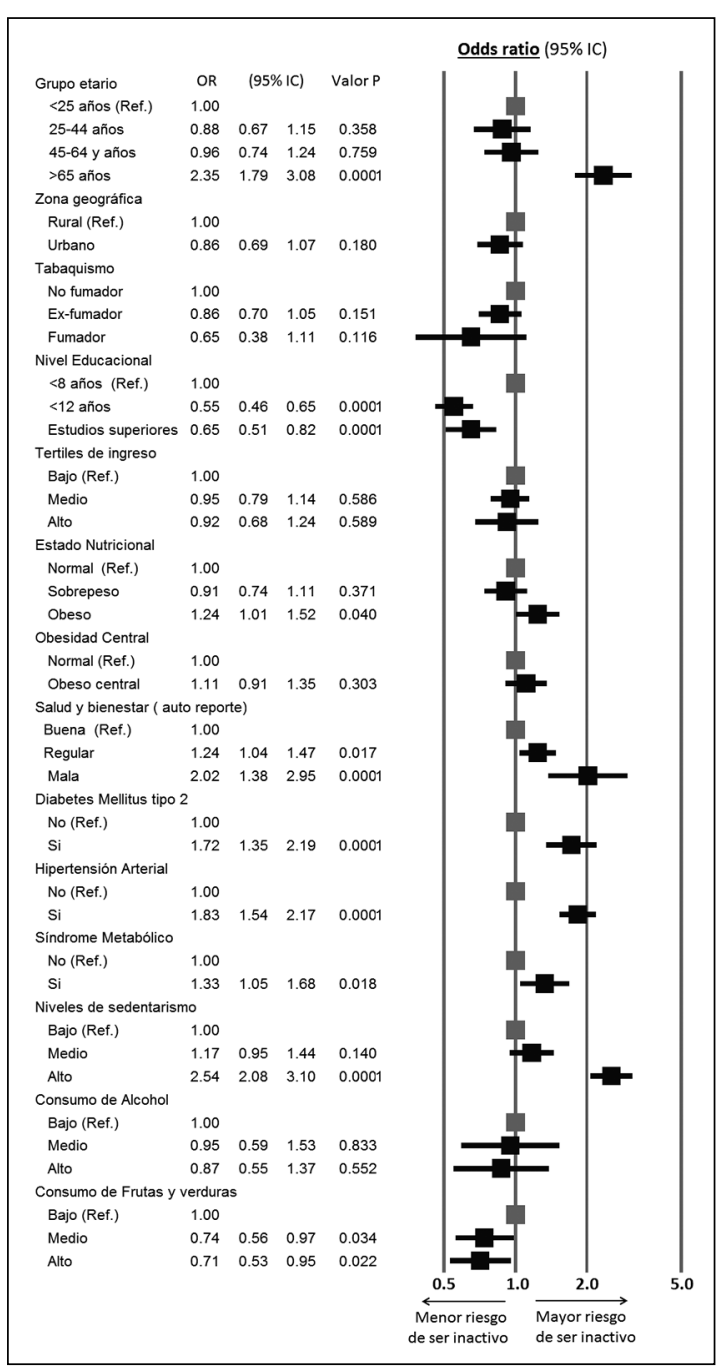

Figura 3. Factores asociados a inactividad fisica en mujeres de la población chilena. Datos presentados como odds ratio y sus respectivos intervalos de confianza de $95 \%$. Todos los análisis fueron ajustados por edad, educación, zona geográfica, tabaquismo e IMC, a excepción de cuando estas variables fueron utilizadas como variable independente en el modelo estadístico. El grupo de referencia utilizado para cada variable se señala con (Ref.).

\section{Discusión}

Los principales resultados de este estudio entregan evidencia que las mujeres en comparación con los hombres, personas mayores a 65 años de edad en comparación a grupos de menor edad, personas con obesidad y con factores de riesgo cardiovascular (diabéticos, hipertensos y síndrome metabólico, etc.), presentan un mayor riesgo de ser físicamente inactivos. Mientras que factores protectores contra la inactividad física fueron el consumo de frutas y verduras y mayores niveles de escolaridad. Estas asociaciones fueron independientes de edad, sexo, zona geográfica, educación, tabaquismo e IMC. Si bien estudios previos han estudiado la asociación entre actividad física y factores de riesgo cardiovascular o los niveles de actividad física laboral y de tiempo libre según factores socio-demográficos en población chilena este es el primer estudio a nivel nacional que presenta evidencia en relación con factores asociados a inactividad física independientes de factores confundidores. Las implicaciones de estos hallazgos podrían ser de utilidad para definir y aplicar más efectivamente iniciativas de intervención orientadas a promover la AF en Chile.

Estos resultados concuerdan con estudios previos como el estudio liderado por Bauman y cols., que reportó que la asociación entre factores socio-demográficos, estilos de vida y de salud con el riesgo de ser físicamente inactivo varía según la población, principalmente según el nivel de ingreso per cápita, como también el desarrollo económico de cada país ${ }^{18}$. Este es un elemento importante que debe ser considerado, ya que factores asociados a inactividad física podrían ser completamente diferentes entre un país desarrollado y uno en vías de serlo $^{19}$. Es importante considerar que los factores de riesgo identificados en este estudio permiten detectar factores asociados a inactividad física que son atingentes a nuestra realidad.

En concordancia con lo expuesto, estos resultados podrían ser de utilidad para mejorar y personalizar las intervenciones y políticas públicas de AF establecida por el estado. Si bien, se sabe que las intervenciones generalizadas tienen un efecto limitado en incrementar los niveles de AF de la población ${ }^{20,21}$, dicho efecto se optimizaría si fueran diseñadas para grupos de individuos que presenten un mayor riesgo de ser inactivos, como lo son personas mayores de 65 años, con nivel educacional equivalente a nivel básico, personas con obesidad y enfermedades cardiovasculares, $o$ aquellos con niveles medios o altos de conducta sedentaria. Esta estrategia al estar focalizada en los grupos de mayor riesgo resultaría más efectiva, para que estas personas modifiquen sus estilos de vida a partir de la incorporación de la actividad 
física en forma cotidiana ${ }^{22-25}$. Nuestros resultados también muestran que personas con mayor nivel educacional y aquellos que reportaron mayor consumo de frutas y verduras presentan un menor odds de ser físicamente inactivos. Estos resultados, están en la dirección esperada, ya que personas con mayor nivel educacional tiene una menor prevalencia de enfermedades cardiovasculares y presentan estilos de vida más saludables que personas con nivel de escolaridad baja. El consumo de frutas y verduras, sería en este caso un marcador de estilo de vida saludable, y que está relacionado a un mayor nivel de escolaridad.

Si bien los resultados reportados por este estudio son representativos para la población chilena, es importante considerar que debido a su diseño observacional de corte transversal no se puede establecer una relación de causa-efecto. Por consiguiente, son necesarios futuros estudios experimentales para responder si intervenciones personalizadas a estos grupos que presenten un mayor riesgo de ser físicamente inactivos, son un medio efectivo para incrementar los niveles de actividad física de la población ${ }^{22}$. Dentro de las limitantes del estudio se encuentra el auto-reporte de actividad física, el cual presenta una baja correlación con metodología objetiva para la medición de actividad física, como lo es acelerometría ${ }^{13,26}$. Por ende, las asociaciones observadas en este estudio podrían estar sesgadas por la sobre estimación de actividad física realizada, principalmente en personas con estilos de vida no saludable, con patologías existentes y aquellas con sobrepeso $\mathrm{u}$ obesidad ${ }^{13,26}$. También es importante tener en cuenta el posible efecto de "causalidad inversa", ya que la identificación de factores protectores o de riesgo para ser físicamente inactivo podrían estar influenciadas por el estado de salud de la población. No obstante, al realizar análisis de sensibilidad y remover a personas que declararon tener diabetes, hipertensión, o síndrome metabólico, los resultados de este estudio no cambiaron la dirección y magnitud de su asociación (estos resultados no fueron reportados en este estudio). Sin embargo, debido al diseño transversal de la ENS 2009-2010 es imposible remover completamente el efecto de causalidad inversa, por ende, futuros estudios longitudinales en población chilena, son necesarios para corroborar los resultados en este estudio.

En conclusión, los resultados de este estudio aportan nueva evidencia en relación a factores de riesgo asociados a inactividad física en Chile, los cuales podrían ser de utilidad para el diseño e implementación de futuras intervenciones o políticas públicas para incrementar los niveles de AF de la población. Estas políticas o intervenciones podrían personalizarse en aquellos grupos que tienen una mayor probabilidad de ser inactivos, lo que podría ayudar a cumplir con las metas sanitarias planteadas para disminuir los niveles de inactividad física y riesgo asociado a enfermedades cardiovasculares $^{12}$.

Agradecimientos: Se agradece de manera especial a todos los participantes de la ENS 2009-10, como también al equipo profesional de la Escuela de Salud Pública, de la Facultad de Medicina de la Pontificia Universidad Católica de Chile, quienes desarrollaron y aplicaron la Encuesta Nacional de Salud y al Ministerio de Salud del Gobierno de Chile.

\section{Referencias}

1. Lee IM, Shiroma EJ, Lobelo F, Puska P, Blair SN, Katzmarzyk PT, et al. Effect of physical inactivity on major non-communicable diseases worldwide: an analysis of burden of disease and life expectancy. Lancet 2012; 380 (9838): 219-29. doi: 10.1016/s0140-6736(12)61031-9.

2. WHO. Global health risks: mortality and burden of disease attributable to selected major risks. WHO, 2009. Available: http://www.who.int/healthinfo/global_burden_disease/GlobalHealthRisks_report_full.pdf

3. Celis-Morales C, Lyall DM, Anderson J, Pell JP, Sattar N, Gill J. The association between physical activity and risk of mortality is modulated by grip strength and cardiorespiratory fitness: evidence from 498,135 UK-Biobank participants. European Heart Journal 2016; 38 (2): 11622. doi: 10.1093/eurheartj/ehw249

4. Ekelund U, Steene-Johannessen J, Brown WJ, Fagerland MW, Owen N, Powell KE, et al. Does physical activity attenuate, or even eliminate, the detrimental association of sitting time with mortality? A harmonised meta-analysis of data from more than 1 million men and women 2016; 388 (10051): [1302-10 pp.].

5. Celis-Morales CA, Lyall DM, Welsh P, Anderson J, Steell L, Guo Y, et al. Association between active commuting and incident cardiovascular disease, cancer, and mortality: prospective cohort study. BMJ 2017; 357. doi:

6. García-Hermoso A, Saavedra JM, Ramírez-Velez R, 
Ekelund U, Del Pozo-Cruz B. Reallocating sedentary time to moderate-to-vigorous physical activity but not to light-intensity physical activity is effective to reduce adiposity among youths: a systematic review and meta-analysis. Obesity reviews : an official journal of the International Association for the Study of Obesity. 2017. doi: 10.1111/obr.12552.

7. MINSAL. Encuesta Nacional de Salud 2009-2010. Chile: Ministerio de Salud, 2010. Available: http://web.minsal. $\mathrm{cl} /$ portal/url/item/bcb03d7bc28b64dfe0400101650 12d23.pdf

8. Giles-Corti B, Vernez-Moudon A, Reis R, Turrell G, Dannenberg AL, Badland $\mathrm{H}$, et al. City planning and population health: a global challenge. Lancet 2016. doi: 10.1016/S0140-6736(16)30066-6.

9. Steell L, Garrido-Méndez A, Petermann F, Díaz-Martínez X, Martínez MA, Leiva AM, et al. Active commuting is associated with a lower risk of obesity, diabetes and metabolic syndrome in Chilean adults. J Public Health (Oxf) 2017; 8 (1): 1-9. doi: https://doi.org/10.1093/ pubmed/fdx092.

10. Díaz-Martínez X, Steell L, Martínez MA, Leiva AM, Salas-Bravo C, Labraña AM, et al. Higher levels of self-reported sitting time is associated with higher risk of type 2 diabetes independent of physical activity in Chile. J Public Health (Oxf) 2017; 1-7. doi: https://doi. org/10.1093/pubmed/fdx091.

11. Celis-Morales C, Salas C, Alduhishy A, Sanzana R, Martínez M, Leiva A, et al. Socio-demographic patterns of physical activity and sedentary behaviour in Chile: results from the National Health Survey 2009-2010. J Public Health (Oxf) 2016; 38 (2): e98-e105. doi:

12. Celis-Morales C, Salas C, Alvarez C, Aguilar Farias N, Ramírez Campillos R, Leppe J, et al. Higher physical activity levels are associated with lower prevalence of cardiovascular risk factors in Chile. Rev Med Chile 2015; 143 (11): 1435-43.

13. Celis-Morales CA, Pérez-Bravo F, Ibáñez L, Salas C, Bailey ME, Gill JM. Objective vs self-reported physical activity and sedentary time: effects of measurement method on relationships with risk biomarkers. Plos One 2012; 7 (5): e36345. doi: 10.1371/journal.pone.0036345.

14. Celis-Morales CA, Perez-Bravo F, Ibanes L, Sanzana R, Hormazabal E, Ulloa N, et al. Insulin Resistance in Chileans of European and Indigenous Descent: Evidence for an Ethnicity x Environment Interaction. Plos One 2011; 6 (9). doi: 10.1371/journal.pone.0024690.

15. Alberti KG, Eckel RH, Grundy SM, Zimmet PZ, Cleeman JI, Donato KA, et al. Harmonizing the Metabolic Syndrome A Joint Interim Statement of the International Diabetes Federation Task Force on Epidemio- logy and Prevention; National Heart, Lung, and Blood Institute; American Heart Association; World Heart Federation; International Atherosclerosis Society; and International Association for the Study of Obesity. Circulation 2009; 120 (16): 1640-5. doi: 10.1161/circulationaha.109.192644.

16. WHO. Global Physical Activity Questionnaire: GPAQ version 2.0. World Health Organization, 2009. 2009. Report No. Available: http://www.who.int/chp/steps/ resources/GPAQ_Analysis_Guide.pdf.

17. WHO. Global recommendations on physical activity for health. World Health Organization, 2010. Available: http://www.who.int/dietphysicalactivity/publications/9789241599979/en/.

18. Bauman AE, Reis RS, Sallis JF, Wells JC, Loos RJ, Martin BW, et al. Correlates of physical activity: why are some people physically active and others not? Lancet 2012; 380 (9838): 258-71. doi: 10.1016/S0140-6736(12)607351.

19. Kohl HW 3rd, Craig CL, Lambert EV, Inoue S, Alkandari JR, Leetongin G, et al. The pandemic of physical inactivity: global action for public health. Lancet 2012; 380 (9838): 294-305. doi: 10.1016/s0140-6736(12)60898-8.

20. Heath GW, Parra DC, Sarmiento OL, Andersen LB, Owen N, Goenka S, et al. Evidence-based intervention in physical activity: lessons from around the world. Lancet 2012; 380 (9838): 272-81. doi: 10.1016/S01406736(12)60816-2.

21. Pratt M, Sarmiento OL, Montes F, Ogilvie D, Marcus $\mathrm{BH}$, Pérez LG, et al. The implications of megatrends in information and communication technology and transportation for changes in global physical activity. Lancet 2012; 380 (9838): 282-93. doi: 10.1016/S01406736(12)60736-3.

22. Celis-Morales C, Lara J, Mathers JC. Personalising nutritional guidance for more effective behaviour change. The Proceedings of the Nutrition Society 2015; 74 (2): 130-8. doi: 10.1017/S0029665114001633

23. Celis-Morales C, Livingstone KM, Marsaux CFM, Macready AL, Fallaize R, O’Donovan CB, et al. Effect of personalized nutrition on health-related behaviour change: evidence from the Food4me European randomized controlled trial. Int J Epidemiol 2017; 46 (2): 578-88. doi: 10.1093/ije/dyw186

24. Marsaux CFM, Celis-Morales C, Livingstone KM, Fallaize R, Kolossa S, Hallmann J, et al. Changes in Physical Activity Following a Genetic-Based Internet-Delivered Personalized Intervention: Randomized Controlled Trial (Food4Me). J Med Internet Res 2016; 18 (2): e30. doi: 10.2196/jmir.5198.

25. Marsaux CFM, Celis-Morales C, Fallaize R, Macready 
AL, Kolossa S, Woolhead C, et al. Effects of a Web-Based Personalized Intervention on Physical Activity in European Adults: A Randomized Controlled Trial. J Med Internet Res 2015; 17 (10): e231. doi: 10.2196/ jmir.4660.
26. Aguilar-Farías N, Leppe Zamora J. Is a single question of the Global Physical Activity Questionnaire (GPAQ) valid for measuring sedentary behaviour in the Chilean population? J Sports Sci 2017; 35 (16): 1652-7. doi: 10.1080/02640414.2016.1229010. 